\title{
MicroRNA-769-3p inhibits tumor progression in glioma by suppressing ZEB2 and inhibiting the Wnt/ $\beta$-catenin signaling pathway
}

\author{
KAI WANG ${ }^{1}$, SHASHA YANG ${ }^{2}$, YISHEN GAO ${ }^{1}$, CAIHONG ZHANG $^{3}$ and QIANGBO SUI $^{1}$ \\ Departments of ${ }^{1}$ Neurosurgery, ${ }^{2}$ Burns and ${ }^{3}$ Ultrasound, Weihai Central Hospital, Weihai, Shandong 264400, P.R. China
}

Received June 26, 2019; Accepted November 1, 2019

DOI: $10.3892 / \mathrm{ol} .2019 .11135$

\begin{abstract}
Accumulating evidence suggests the crucial role of microRNAs (miRNAs) in human cancers. The present study aimed to investigate the clinical and functional roles of miR-769-3p in glioma, as well as the underlying molecular mechanisms. Reverse transcription-quantitative polymerase chain reaction (RT-qPCR) was used to determine the expression levels of miR-769-3p in glioma tissues and cells. Receiver operating characteristic (ROC) curve analysis was applied to calculate the diagnostic value of miR-769-3p. The 5-year survival rate of patients was calculated using Kaplan-Meier analysis and Cox regression analysis. Cell experiments were used to investigate the functional role of miR-769-3p in glioma. The gene target of miR-769-3p was predicted by TargetScan. Changes in the levels of Wnt signaling-related proteins were measured by western blotting. miR-769-3p was significantly downregulated in glioma tissues and serum, as well as in glioma cell lines $(\mathrm{P}<0.001)$. miR-769-3p expression was significantly associated with the World Health Organization grade and Karnofsky performance score. The ROC curves demonstrated that serum miR-769-3p level reliably distinguished patients with glioma from healthy individuals. High tissue miR-769-3p expression predicted poor overall survival in patients with glioma $(\log$-rank $\mathrm{P}=0.001)$ and was identified as an independent prognostic factor. In addition, zinc finger E-box binding homeobox 2 (ZEB2) was demonstrated to be a direct target of miR-769-3p in glioma cells using a luciferase assay. miR-769-3p upregulation suppressed the activity of the $\mathrm{Wnt} / \beta$-catenin signaling pathway in glioma cells. In conclusion, miR-769-3p may serve as a diagnostic and prognostic biomarker in patients with glioma and target ZEB2 to inhibit tumor progression via the $\mathrm{Wnt} / \beta$-catenin signaling pathway. miR-769-3p may be a novel therapeutic target for the treatment of glioma.
\end{abstract}

Correspondence to: Professor Qiangbo Sui, Department of Neurosurgery, Weihai Central Hospital, 3 West Mishan East Road, Weihai, Shandong 264400, P.R. China

E-mail: chenwen0376493@163.com

Key words: microRNA-769-3p, zinc finger E-box binding homeobox 2, progression, Wnt/ $\beta$-catenin pathway, glioma

\section{Introduction}

Glioma is the most common form of brain cancer (1), and the prognosis of glioma is relatively poor (2). Glioma cells infiltrate the healthy tissues surrounding the main tumor mass, limiting the survival of patients with glioma (3). Despite advances in technology and medicine, the incidence and mortality rates of glioma still continue to increase (4). Furthermore, the incidence of glioma is $\sim 22 / 100,000$ people, and the 5-year survival rate is $\sim 10 \%$ (5). In recent years, dysregulation of oncogenes and tumor suppressor genes during glioma progression has been observed (6). Glioma treatment outcomes may be improved by the identification of potential therapeutic targets based on an improved understanding of the molecular signaling pathways involved in glioma progression.

MicroRNAs (miRNAs) are a class of small and non-coding RNA molecules that are 22 nucleotides long. miRNAs serve as post-transcriptional regulators for gene expression by targeting the 3'-untranslated region (3'UTR) of their target mRNAs, resulting in mRNA degradation or inhibition of protein translation (7). miRNAs have been widely reported to be involved in the progression of various types of cancer, as they can function as oncogenes or tumor suppressors $(8,9)$. Recently, certain aberrantly expressed miRNAs have been identified in glioma that serve crucial roles in tumor progression. For instance, downregulation of miR-449 was detected in glioma tissues and demonstrated to be involved in the tumor progression by targeting flotillin 2 , which was associated with poor prognosis in patients with glioma (10). Upregulation of miR-6807-3p was observed in glioma specimens; miR-6807-3p promoted tumor progression and suppressed apoptosis by targeting dachshund family transcription factor 1 (11). In addition, a microRNA microarray assay indicated that miR-769-3p was differentially expressed in glioma tissues compared with matched normal tissues (12), and the dysregulation of miR-769-3p has been reported in several types of human cancer, such as colorectal cancer and hepatocellular carcinoma $(13,14)$. A recent study on the role of the p53 R273H mutation in the tumor microenvironment has revealed that miR-769-5p is closely associated with p53, suggesting its potential role in pulmonary metastasis (15). However, the exact clinical and functional roles of miR-769-3p in glioma have not been previously investigated.

The present study aimed to examine the expression levels and clinical roles of miR-769-3p in patients with glioma and 
glioma cell lines and to further explore the potential underlying molecular mechanisms.

\section{Materials and methods}

Patients and sample collection. A total of 113 patients, who were pathologically diagnosed with glioma and underwent resection of the primary tumor between August 2010 and January 2012 in Weihai Central Hospital (Weihai, China), were enrolled in the study. Paired samples of glioma and adjacent healthy tissues ( $>3 \mathrm{~cm}$ from cancer tissues) were collected. All tissue samples were immediately snap-frozen in liquid nitrogen and stored at $-80^{\circ} \mathrm{C}$ for future use. In addition, preoperative serum samples were collected from the patients, and 95 additional serum samples were collected from healthy volunteers undergoing routine physical examination at the same hospital to serve as the control group. The healthy control group had a similar age and sex ratio to the patient group and had no history of cancer. The blood samples were centrifuged at $1,000 \mathrm{x} \mathrm{g}$ for $20 \mathrm{~min}$ at $4^{\circ} \mathrm{C}$ within $1 \mathrm{~h}$ after collection, and the plasma was stored at $-80^{\circ} \mathrm{C}$ until further processing. None of the patients received radiotherapy or chemotherapy prior to sample collection. The present study was approved by the Ethics Committee of Weihai Central Hospital, and written informed consent was obtained from each participant.

Cell culture and transfection. Human glioma cell lines LN-229, A-172, T98G and SHG-44, as well as normal human astrocytes (NHAs) were obtained from the American Type Culture Collection (Manassas). All cell lines were cultured in Dulbecco's modified Eagle's medium (DMEM; Thermo Fisher Scientific, Inc.) containing 10\% fetal bovine serum (FBS; Thermo Fisher Scientific, Inc.) and maintained in a humidified incubator at $37^{\circ} \mathrm{C}$ with $5 \% \mathrm{CO}_{2}$.

The miR-769-3p mimic, miR-769-3p inhibitor and the corresponding negative controls (mimic NC and inhibitor NC) were synthesized and purified by Shanghai GenePharma Co., Ltd. The sequence were as follows: miR-769-3p mimic, 5'-UUGGUU CUGGGGCCUCUAGGGUC-3'; mimic NC, 5'-UUCUCC GAACGUGUCACGUTT-3'; miR-769-3p inhibitor, 5'-GAC CCUAGAGGCCCCAGAACCAA-3'; inhibitor NC, 5'-CAG UACUUUUGUGUAGUACAA-3'. Lipofectamine ${ }^{\circledR} 3000$ reagent (Invitrogen; Thermo Fisher Scientific, Inc.) was used to transfect the miRNAs (100 nM) into glioma cells (A-172 and SHG-44) according to the manufacturer's protocol. Cells were harvested for subsequent experiments following $48 \mathrm{~h}$ incubation at $37^{\circ} \mathrm{C}$.

$R N A$ extraction and reverse transcription-quantitative polymerase chain reaction ( $R T-q P C R)$. Total RNA was extracted from the tissue or serum samples and cells using TRIzol ${ }^{\circledR}$ Reagent (Invitrogen; Thermo Fisher Scientific, Inc.) according to the manufacturer's protocol. RNA quality and concentration were assessed using a NanoDrop ND1000 Spectrophotometer (Thermo Fisher Scientific, Inc.). RNA was reverse transcribed to cDNA using a miRcute miRNA cDNA first-strand synthesis kit (Tiangen Biotech Co., Ltd.) according to the manufacturer's instructions. SYBR-Green I Master Mix kit (Invitrogen; Thermo Fisher Scientific, Inc.) was used to perform qPCR. U6 was used as an internal control, and the relative expression levels were estimated using the $2^{-\Delta \Delta \mathrm{Cq}}$ method (16). The primer sequences used were: miR-769-3p forward, 5'-TCGGCAGGC TGGGATCTCCGGGG-3' and reverse, 5'-GTGCAGGGT CCGAGGT-3'; U6 forward, 5'-CTCGCTTCGGCAGCACA-3' and reverse, 5'-AACGCTTCACGAATTTGCGT-3'.

MTT assay. The proliferation of glioma cells was determined using MTT assay. Once A-172 and SHG-44 cells were transfected with miR-769-3p mimic or inhibitor, or their negative controls for $48 \mathrm{~h}$, cells were harvest for subsequent experiments. The transfected cells were seeded in 96-well plates $\left(1 \times 10^{4}\right.$ cells/well) and incubated in a humidified incubator with $5 \% \mathrm{CO}_{2}$ at $37^{\circ} \mathrm{C}$ for $16-48 \mathrm{~h}$. Subsequently, $20 \mu \mathrm{l} 5 \% \mathrm{MTT}$ solution was added into each well, followed by an additional 4-h incubation with $5 \% \mathrm{CO}_{2}$ at $37^{\circ} \mathrm{C}$ in the dark. The medium was discarded, and $100 \mu \mathrm{l}$ dimethyl sulfoxide was added to stop the reaction. Cell proliferation was estimated by measuring the optical density at $490 \mathrm{~nm}$ using a microplate reader.

Transwell assay. Cell migration and invasion were analyzed using a Transwell chambers with a pore size of $8 \mu \mathrm{m}$ (Corning, Inc.). For the invasion analysis, the membranes were pre-treated with Matrigel (Corning, Inc.), whereas uncoated membranes were used for the migration assay. Once A-172 and SHG-44 cells were transfected with miR-769-3p mimic or inhibitor, or their negative controls for $48 \mathrm{~h}$, the cells $\left(1 \times 10^{5}\right)$ were collected, resuspended in $200 \mu 1$ serum-free DMEM and seeded into the upper chamber, whereas $300 \mu$ I DMEM containing $10 \%$ FBS was added into the lower chamber to act as a chemoattractant. Following incubation for $48 \mathrm{~h}$ at $37^{\circ} \mathrm{C}$, cells remaining on the upper surface of the membranes were removed with a cotton swab. The cells that had migrated into the lower chambers were fixed in $4 \%$ paraformaldehyde at room temperature for $30 \mathrm{~min}$ and stained with $0.5 \%$ crystal violet at room temperature for $30 \mathrm{~min}$. The number of migrated cells was counted using a light microscope (magnification, x200; Olympus Corporation).

Luciferase reporter assay. A putative binding site in the 3'UTR of zinc finger E-box binding homeobox 2 (ZEB2) was identified for miR-769-3p by TargetScan (http://www.targetscan.org) analysis. The luciferase reporter gene assay was performed to determine whether ZEB2 was indeed a target gene of miR-769-3p. Briefly, the miR-769-3p-binding site in the ZEB2 3'UTR (wild type or mutant) was cloned downstream of the firefly luciferase gene in a pGL3-promoter vector. The luciferase activity was measured using a Dual-Luciferase Reporter Assay System (Promega Corporation). Renilla luciferase activity was used for normalization.

Western blot assay. Proteins were extracted using RIPA lysis buffer (Beyotime Institute of Biotechnology), separated by SDS-PAGE and transferred onto PVDF membranes (EMD Millipore). The membranes were blocked with 5\% non-fat dried milk and incubated with primary antibodies. The antibodies targeting $\beta$-catenin and cyclin D1 were purchased from Cell Signaling Technology, Inc. Quantitative densitometric analysis of the immunoblotting images was performed using ImageJ software (version 1.8.0; National Institutes of Health). 

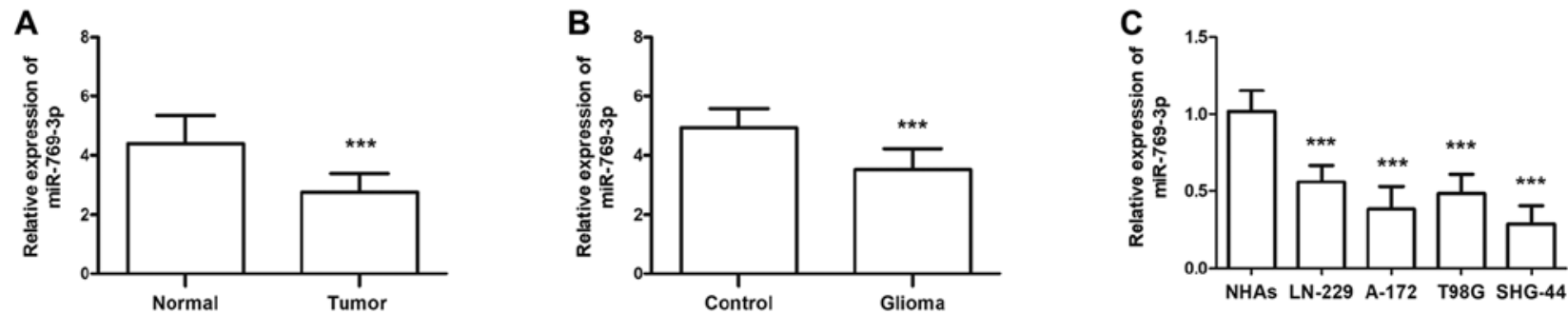

Figure 1. Expression of miR-769-3p measured by reverse transcription-quantitative PCR in glioma tissues, serum samples and cell lines. (A) Tissue expression level of miR-769-3p was significantly downregulated in glioma compared with adjacent healthy tissues. (B) Serum expression level of miR-769-3p was significantly downregulated in patients with glioma compared with healthy control subjects. (C) The expression of miR-769-3p was lower in glioma cell lines LN-229, A-172, T98G and SHG-44 compared with that in normal glioma cells. ${ }^{* * *} \mathrm{P}<0.001$ vs. control. miR, microRNA.

Statistical analysis. Data were analyzed using SPSS 18.0 (SPSS, Inc.) and GraphPad Prism 5.0 (GraphPad Software, Inc.). Data are expressed as the mean \pm SD. Student's t-test was used to analyze the differences between two groups, whereas one-way ANOVA followed by Tukey's multiple comparison test was applied to analyze multiple groups for statistical significance. The associations between miR-769-3p expression and clinicopathological characteristics of patients with glioma were determined by $\chi^{2}$ test. Receiver operating characteristic (ROC) curve was used to assess the diagnostic specificity and sensitivity of miR-769-3p levels. The 5-year survival rate of patients was calculated by Kaplan-Meier analysis with log-rank test. Cox regression analysis was used to further determine the prognostic value of miR-769-3p levels in patients with glioma. $\mathrm{P}<0.05$ was considered to indicate a statistically significant difference.

\section{Results}

Expression levels of miR-769-3p in glioma miR-769-3p expression levels were initially detected in 113 paired glioma and adjacent healthy tissues. The results of the RT-qPCR analysis demonstrated that miR-769-3p levels were significantly decreased in glioma compared with adjacent healthy tissues $(\mathrm{P}<0.001$; Fig. 1A). Similar results were observed for patient serum miR-769-3p levels compared with serum samples from healthy individuals $(\mathrm{P}<0.001$; Fig. 1B). The expression levels of miR-769-3p were also investigated in four glioma cell lines (LN-229, A-172, T98G and SHG-44); the results revealed that compared with the levels in normal NHAs, miR-769-3p expression levels were significantly lower in glioma cell lines (all $\mathrm{P}<0.001$; Fig. 1C).

Association between miR-769-3p expression and clinicopathological characteristics of patients with glioma. To investigate the association of miR-769-3p levels with the clinicopathological characteristics of patients with glioma, the patients were classified into low and high expression groups according to the mean value of miR-769-3p levels in glioma tissues or serum (Table I). The $\chi^{2}$ test was used to identify the differences in clinicopathological characteristics between the two groups. The results demonstrated that miR-769-3p levels in the serum and glioma tissues were significantly associated with the World Health Organization (WHO) grade $(\mathrm{P}<0.01)(17)$ and Karnofsky performance score (KPS; P $<0.05)$ (18). No similar results were observed for other clinicopathological parameters, including age, sex and tumor size (all $\mathrm{P}>0.05$; Table I).
ROC analysis of the diagnostic value of serum miR-769-3p level for glioma. The ROC curve is a graphical representation that reflects the association between the sensitivity and specificity of a laboratory test. The ROC curve analysis revealed that serum miR-769-3p levels could reliably distinguish patients with glioma from healthy individuals with an area under the curve value of 0.925 . At the optimal cut-off value of 4.085 , the sensitivity and specificity were 90.5 and $80.5 \%$, respectively (Fig. 2A).

Prognostic value of tissue miR-769-3p level in patients with glioma. Kaplan-Meier analysis was used to assess the prognostic value of tissue miR-769-3p level in patients with glioma. The results demonstrated that patients with a high miR-769-3p expression in the glioma tissue exhibited relatively poor overall survival compared with those in the low expression group (log-rank $\mathrm{P}=0.001$; Fig. 2B). In addition, the clinical parameters and tissue miR-769-3p level were included in the multivariate Cox analysis to determine their influence on the overall survival of patients with glioma. The results demonstrated that the tissue miR-769-3p expression level [hazard ratio (HR), 2.556; 95\% $\mathrm{CI}, 1.449-4.508 ; \mathrm{P}=0.001]$ and the WHO grade (HR, 0.544; 95\% CI, 0.314-0.943; $\mathrm{P}=0.030$ ) were independent prognostic factors for glioma (Table II).

Effects of miR-769-3p on cell proliferation, migration and invasion in glioma cells. Since miR-769-3p expression was downregulated to a greater extent in A-172 and SHG-44 cells compared with the other glioma cell lines $(\mathrm{P}<0.001$; Fig. 1B), the two cell lines were used in subsequent experiments. miR-769-3p was overexpressed or downregulated in A-172 and SHG-44 cells by transfection with miR-769-3p mimic or miR-769-3p inhibitor, respectively. The transfection efficiency was determined by RT-qPCR, and the results indicated that transfection with the miR-769-3p mimic resulted in an increase in miR-769-3p expression levels, whereas transfection with the miR-769-3p inhibitor significantly decreased its expression ( $\mathrm{P}<0.01$; Fig. $3 \mathrm{~A})$. The MTT assay results revealed that the overexpression of miR-769-3p significantly inhibited cell proliferation in A-172 and SHG-44 cells, whereas silencing of miR-769-3p enhanced cell proliferation ( $\mathrm{P}<0.05$; Fig. 3B). The results of the Transwell assay demonstrated that in A-172 and SHG-44 cells, the number of migrated cells was significantly lower in the miR-769-3p mimics group compared with that in the mimic $\mathrm{NC}$ group, but higher in the miR-302b inhibitor group compared with that in the inhibitor $\mathrm{NC}$ group ( $\mathrm{P}<0.001$; Fig. 4A and B). Additionally, overexpression 
Table I. Association of tissue and serum miR-769-3p with the clinicopathological characteristics of patients with glioma.

\begin{tabular}{|c|c|c|c|c|c|c|c|}
\hline \multirow[b]{2}{*}{ Characteristic } & \multirow[b]{2}{*}{ Total $(n=113)$} & \multicolumn{2}{|c|}{ Tissue miR-769-3p expression } & \multirow[b]{2}{*}{ P-value } & \multicolumn{2}{|c|}{ Serum miR-769-3p expression } & \multirow[b]{2}{*}{ P-value } \\
\hline & & Low $(\mathrm{n}=69)$ & $\operatorname{High}(\mathrm{n}=44)$ & & Low $(\mathrm{n}=59)$ & High $(\mathrm{n}=54)$ & \\
\hline \multicolumn{8}{|l|}{ Age, years } \\
\hline$\leq 60$ & 60 & 37 & 23 & & 30 & 30 & \\
\hline$>60$ & 53 & 32 & 21 & 0.888 & 29 & 24 & 0.616 \\
\hline \multicolumn{8}{|l|}{ Sex } \\
\hline Male & 59 & 36 & 23 & & 31 & 28 & \\
\hline Female & 54 & 33 & 21 & 0.992 & 28 & 26 & 0.941 \\
\hline \multicolumn{8}{|l|}{ Tumor size, $\mathrm{cm}$} \\
\hline$<5.0$ & 57 & 30 & 27 & & 27 & 30 & \\
\hline$\geq 5.0$ & 56 & 39 & 17 & 0.064 & 32 & 24 & 0.298 \\
\hline \multicolumn{8}{|l|}{ WHO grade } \\
\hline I-II & 58 & 28 & 30 & & 23 & 35 & \\
\hline III-IV & 55 & 41 & 14 & $0.004^{\mathrm{b}}$ & 36 & 19 & $0.006^{\mathrm{b}}$ \\
\hline \multicolumn{8}{|l|}{ KPS } \\
\hline$<80$ & 58 & 41 & 17 & & 37 & 21 & \\
\hline$\geq 80$ & 55 & 28 & 27 & $0.031^{\mathrm{a}}$ & 22 & 33 & $0.011^{\mathrm{a}}$ \\
\hline
\end{tabular}

${ }^{\mathrm{a}} \mathrm{P}<0.05 ;{ }^{\mathrm{b}} \mathrm{P}<0.01$. WHO, World Health Organization; KPS, Karnofsky performance scale; miR, microRNA.

of miR-769-3p significantly reduced the number of invasive cells, whereas silencing of miR-769-3p increased the number of invasive cells ( $\mathrm{P}<0.01$; Fig. $4 \mathrm{C}$ and $\mathrm{D})$.

ZEB2 is a target gene of miR-769-3p in glioma. TargetScan was used to identify the target genes of miR-769-3p and revealed that ZEB2 mRNA contained seven matched nucleotides with miR-769-3p at position 3871-3877 in the 3'UTR (Fig. 5A). The results of the luciferase reporter assay demonstrated that miR-769-3p mimic transfection attenuated the luciferase activity of ZEB2 3'UTR, whereas silencing of miR-769-3p promoted ZEB2 3'UTR luciferase activity; this was not observed when the mutant ZEB2 3'UTR was expressed (Fig. 5B). In addition, RT-qPCR analysis of ZEB2 mRNA expression levels revealed that miR-769-3p overexpression decreased ZEB2 expression, whereas opposite results were observed when miR-769-3p expression was silenced (Fig. 5C).

miR-769-3p is involved in the regulation of the Wnt/ $\beta$-catenin signaling pathway. Western blotting was performed to determine whether miR-769-3p may influence the levels of Wnt signaling-related proteins. As presented in Fig. 6, overexpression of miR-769-3p significantly suppressed the expression of Wnt signaling-related proteins $\beta$-catenin and cyclin D1, whereas silencing of miR-769-3p exhibited the opposite result.

\section{Discussion}

Glioma is the most common primary malignant tumor of the brain (19). The majority of patients with glioma experience recurrence with poor prognosis, leading to challenges in the
Table II. Multivariate Cox regression analysis of miR-769-3p expression and clinicopathological characteristics in patients with glioma.

\begin{tabular}{lccc}
\hline & \multicolumn{3}{c}{ Multivariate analysis } \\
\cline { 2 - 4 } Variable & HR & $95 \%$ CI & P-value \\
\hline $\begin{array}{l}\text { miR-769-3p expression } \\
\text { (low vs. high) }\end{array}$ & 2.556 & $1.449-4.508$ & $0.001^{\mathrm{b}}$ \\
$\begin{array}{l}\text { Age, years }(\leq 60 \text { vs. }>60) \\
\text { Sex (male vs. female) }\end{array}$ & 1.111 & $0.647-1.907$ & 0.703 \\
$\begin{array}{l}\text { Tumor size, cm } \\
(<5.0 \text { vs. } \geq 5.0)\end{array}$ & 0.747 & $0.850-2.548$ & 0.167 \\
$\begin{array}{l}\text { WHO grade } \\
\text { (I-II vs. III-IV) }\end{array}$ & 0.544 & $0.436-1.281$ & 0.289 \\
KPS $(<80$ vs. $>80)$ & 0.696 & $0.398-1.215$ & 0.202 \\
\hline
\end{tabular}

${ }^{\mathrm{a}} \mathrm{P}<0.05 ; \quad{ }^{\mathrm{b}} \mathrm{P}<0.01$. WHO, World Health Organization; KPS, Karnofsky performance scale; miR, microRNA; HR, hazard ratio; $\mathrm{CI}$, confidence interval.

treatment of malignant glioma (20). The common treatment methods for glioma include radiotherapy, chemotherapy and surgery (21). However, due to the specific location of these tumors, the application of radiotherapy and chemotherapy is limited (22). Thus, it is of great significance to identify glioma-related therapeutic targets to improve patient outcomes. miRNAs have been demonstrated to be involved in different types of human cancer, including 

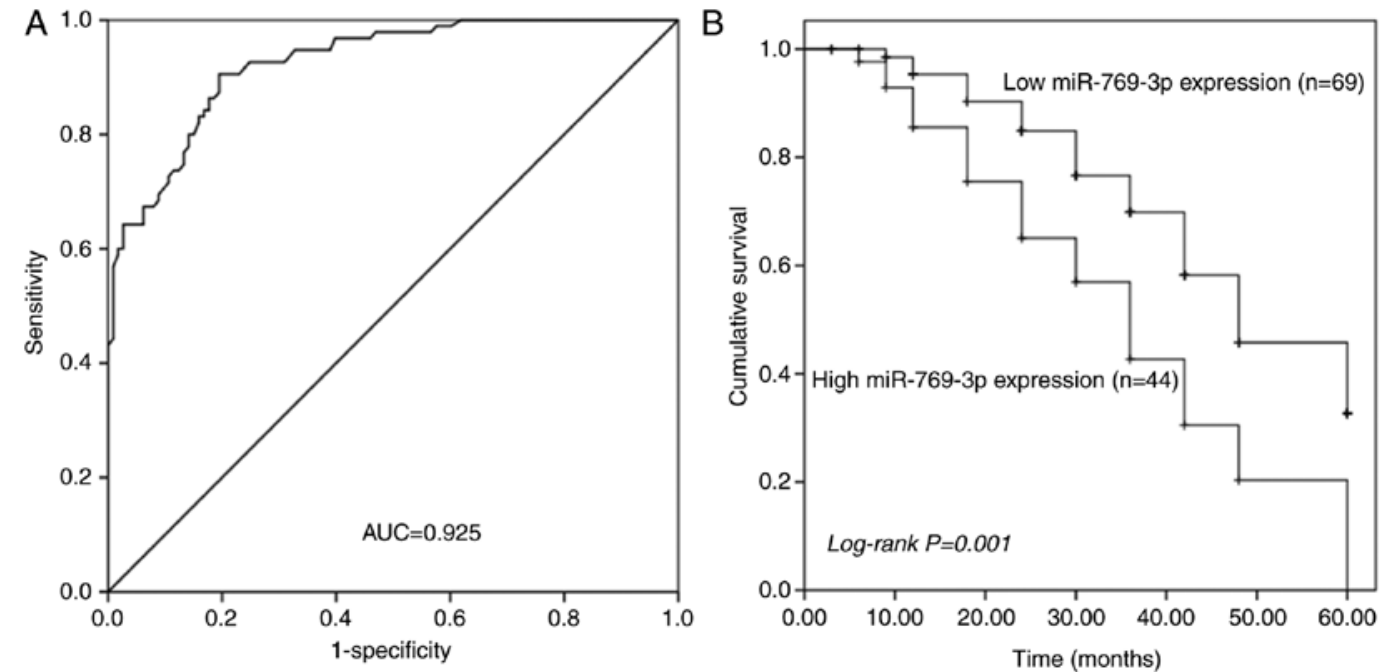

Figure 2. Diagnostic value of miR-769-3p in glioma. (A) The receiver operating characteristic curve analysis of patients with glioma based on the serum miR-769-3p levels demonstrated an AUC value of 0.925, with the sensitivity and specificity of 90.5 and $80.5 \%$, respectively. (B) Survival analysis in patients with glioma based on the tissue expression of miR-769-3p. Patients with high miR-769-3p expression exhibited shorter survival time compared with those with low miR-769-3p expression. AUC, area under the curve; miR, microRNA.

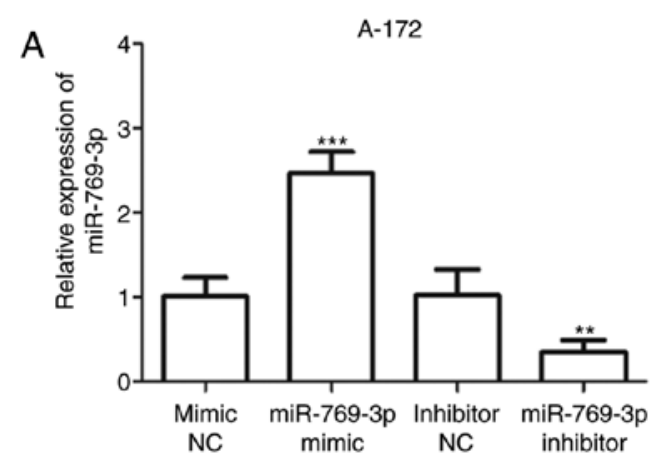

B

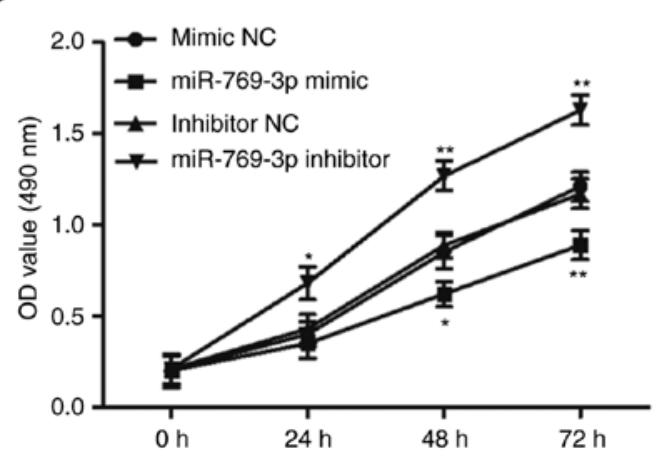

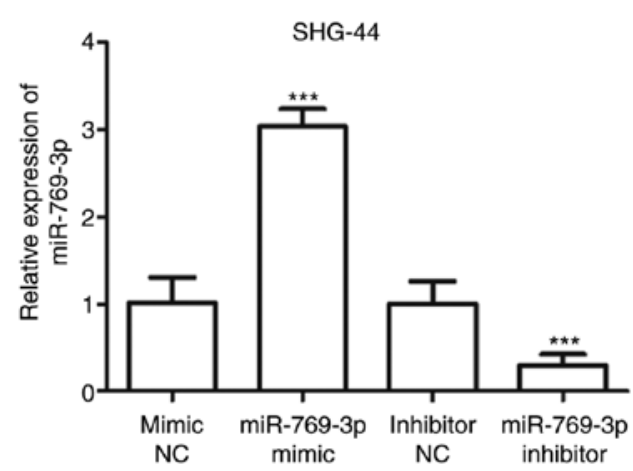

SHG-44

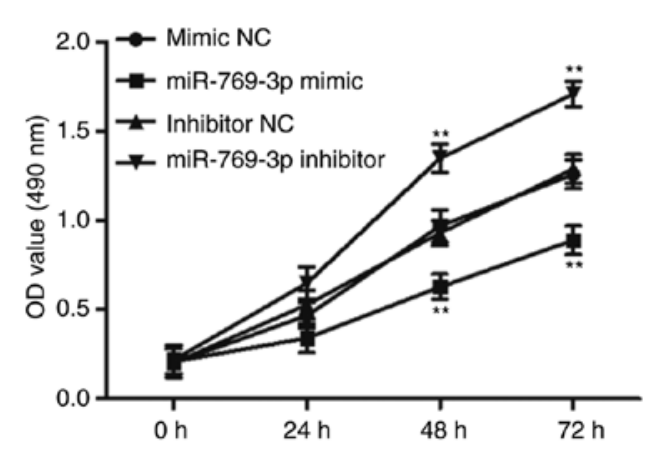

Figure 3. Effects of miR-769-3p on cell proliferation in A-172 and SHG-44 cells. (A) In A-172 and SHG-44 cells, the expression levels of miR-769-3p were significantly increased following miR-769-3p mimic transfection, but decreased following miR-769-3p inhibitor transfection compared with the corresponding NC. (B) Cell proliferation was suppressed by overexpression of miR-769-3p, but promoted by silencing of miR-769-3p in A-172 and SHG-44 cells. "P<0.05, ${ }^{* *} \mathrm{P}<0.01$ and ${ }^{* * * *} \mathrm{P}<0.001$ vs. the corresponding NC. miR, microRNA; NC, negative control.

glioma (23). Certain miRNAs have been identified to be aberrantly expressed in glioma and to be involved in tumor progression (24).

The dysregulation of miR-769-3p has been reported in several types of cancer, such as colorectal cancer and melanoma $(13,25)$. In the present study, the expression level of miR-769-3p was determined to be downregulated in glioma, which was consistent with a previous study (12). Additionally, the present result suggested that miR-769-3p levels were significantly associated with WHO grade and KPS in patients with glioma.As the alteration of miR-769-3p expression in glioma was identified, its clinical value was further examined in glioma diagnosis and prognosis in the present study. The ROC curve analysis revealed that serum miR-769-3p levels could reliably distinguish patients with glioma from healthy individuals. Considering these results, it may be concluded 

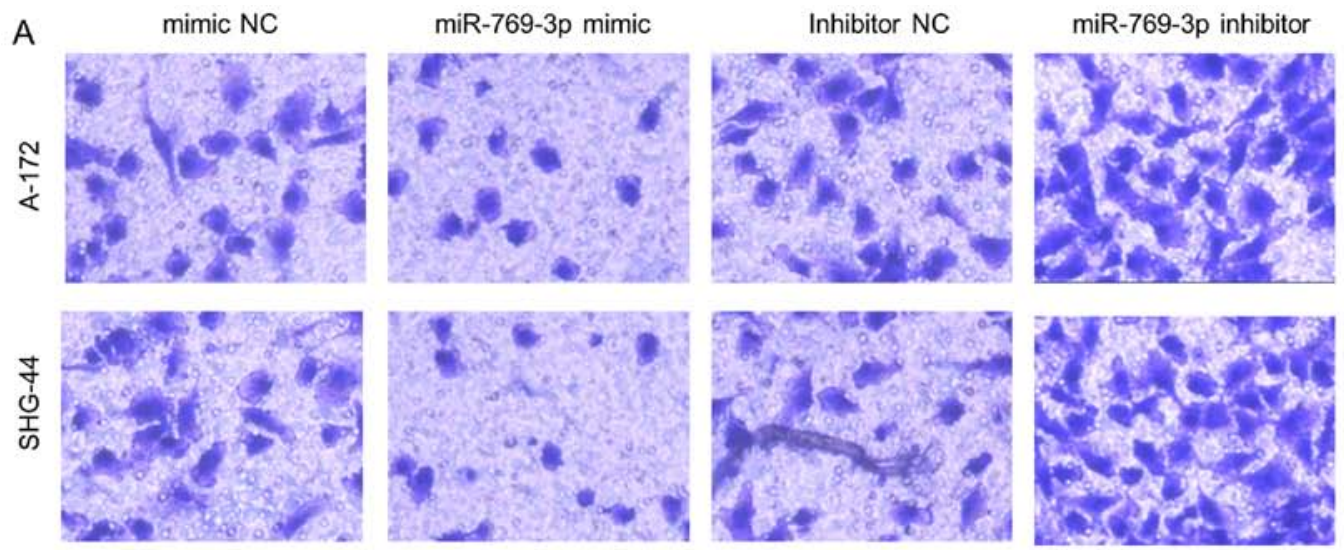

B
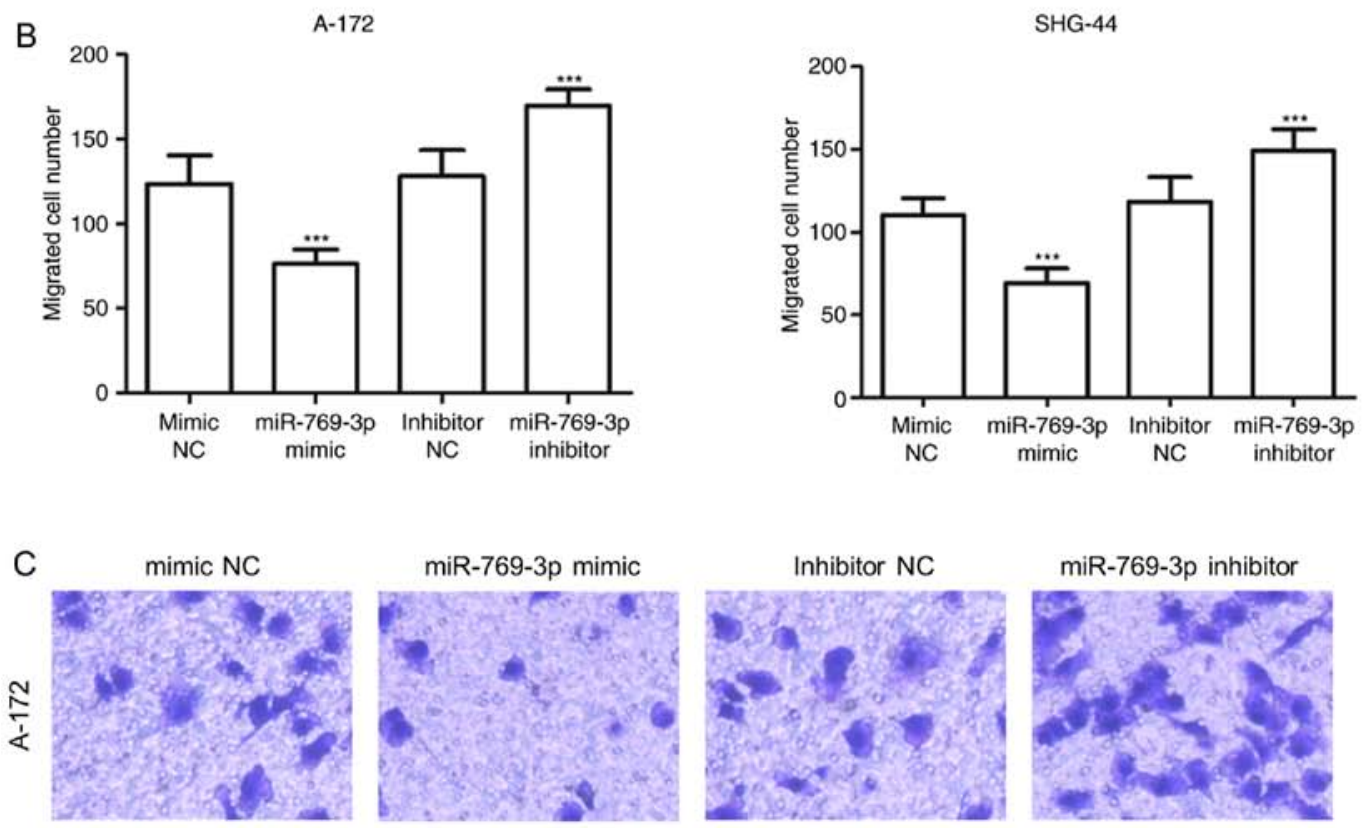

miR-769-3p inhibitor
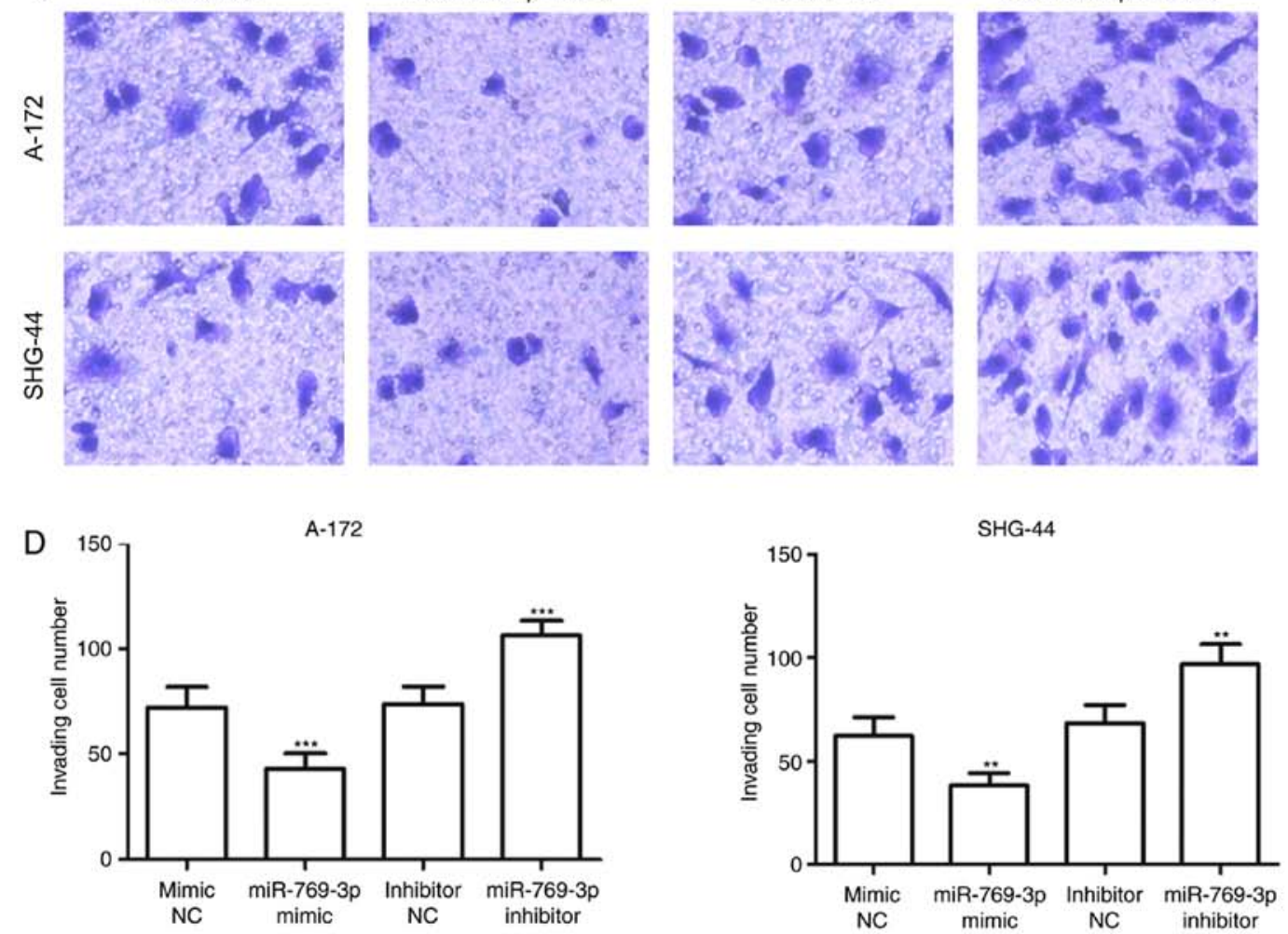

Figure 4. Effects of miR-769-3p on cell migration and invasion in A-172 and SHG-44 cells. (A) Results of migration analysis for A-172 and SHG-44 cells. (B) Overexpression of miR-769-3p inhibited cell migration, whereas miR-769-3p silencing promoted cell migration. (C) Results of invasion analysis for A-172 and SHG-44 cells. (D) Overexpression of miR-769-3p inhibited cell invasion, whereas miR-769-3p silencing promoted cell invasion. ${ }^{* *} \mathrm{P}<0.01$ and ${ }^{* * * *} \mathrm{P}<0.001$ vs. the corresponding NC. miR, microRNA; NC, negative control.

that miR-769-3p may be a tumor suppressor gene and serve as a potential diagnostic factor for glioma. Additionally,
Kaplan-Meier survival analysis indicated that low expression levels of miR-769-3p were significantly associated with poor 


\section{A Position 3871-3877 of ZEB2 3' UTR 5' $\ldots$ UUUUAUUUGACUAUGUGAUCCCAU...}
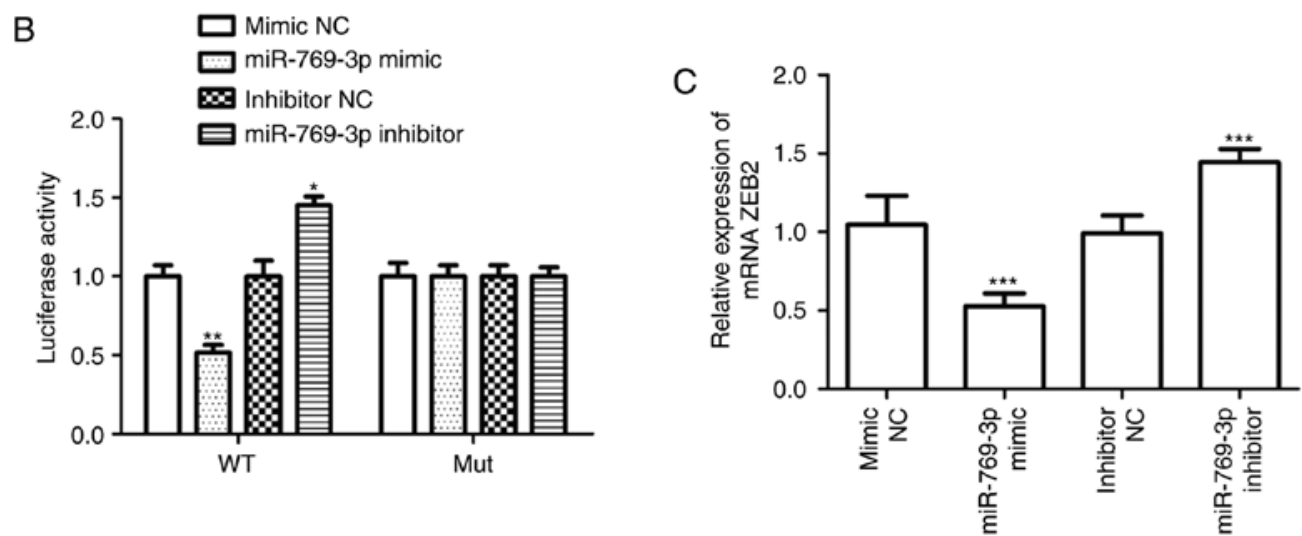

Figure 5. miR-769-3p inhibits ZEB2 expression by binding its 3'UTR. (A) Schematic representation of miR-769-3p and its target sequence within the ZEB2 3'UTR. (B) The luciferase activity of WT or Mut ZEB2 3'UTR in SHG-44 cells transfected with the miR-769-3p mimic or inhibitor. (C) ZEB2 mRNA expression in SHG-44 cells transfected with the miR-769-3p mimic or inhibitor. ${ }^{*} \mathrm{P}<0.05,{ }^{* * *} \mathrm{P}<0.01$ and ${ }^{* * *} \mathrm{P}<0.001$ vs. the corresponding NC. miR, microRNA; UTR, untranslated region; ZEB2, zinc finger E-box binding homeobox 2; WT, wild-type; Mut, mutant; NC, negative control.

A
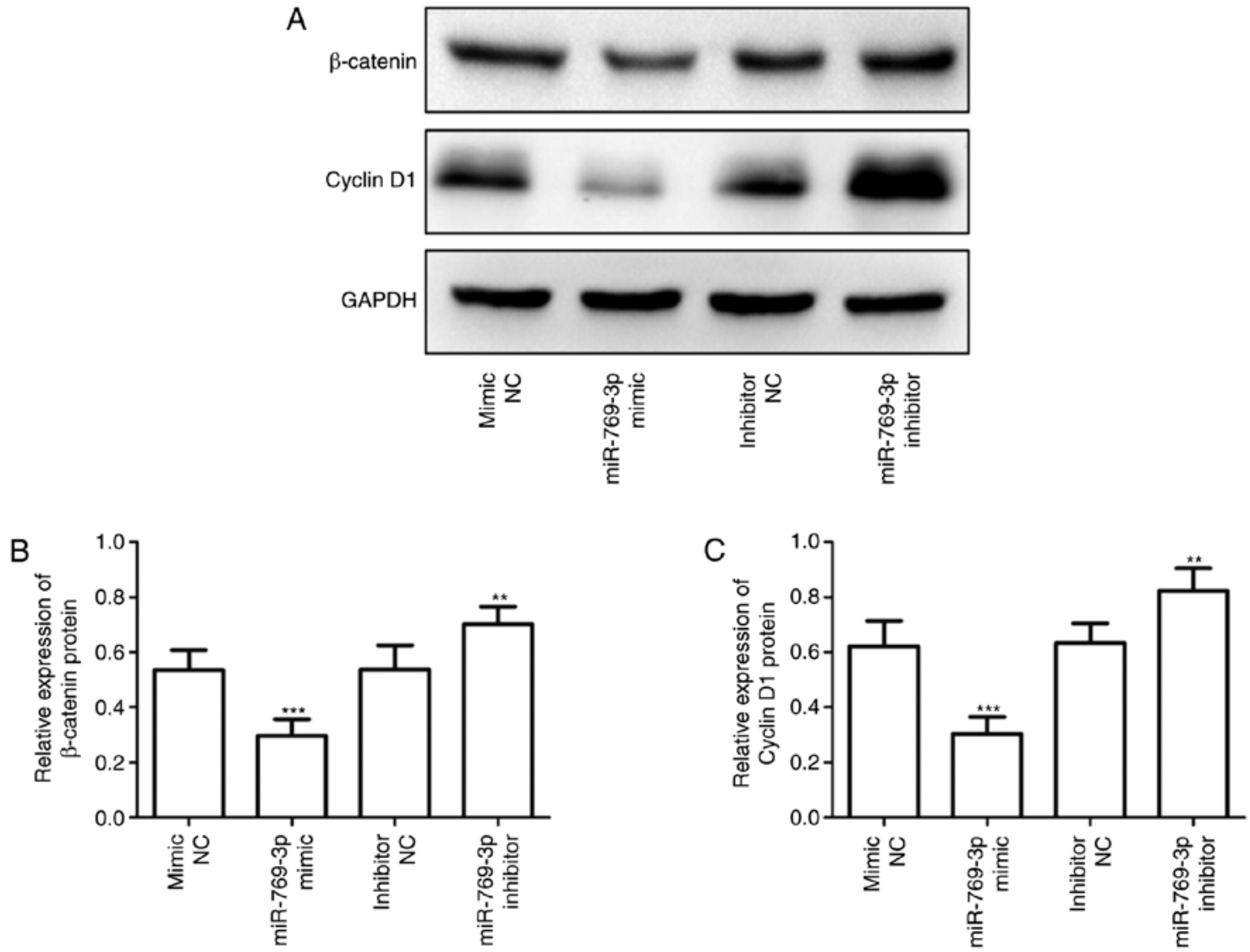

Figure 6. Regulation of the Wnt/ $\beta$-catenin signaling pathway by miR-769-3p. (A) Western blot analysis of $\beta$-catenin and cyclin D1 expression in SHG-44 cells transfected with the miR-769-3p mimic, inhibitor or corresponding NC. (B) Quantification of the western blotting results of $\beta$-catenin expression in SHG-44 cells transfected with the miR-769-3p mimic, inhibitor or corresponding NC. (C) Quantification of the western blotting results of cyclin D1 expression in SHG-44 cells transfected with the miR-769-3p mimic, inhibitor or corresponding NC. ${ }^{* *} \mathrm{P}<0.01$ and ${ }^{* * *} \mathrm{P}<0.001$ vs. the corresponding NC. miR, microRNA; $\mathrm{NC}$, negative control.

overall survival of patients with glioma. Several clinicopathological variables have been previously used to predict prognosis for patients with glioma, such as the WHO grade and KPS score (26). In the present study, the WHO grade and KPS score of the enrolled patients with glioma were analyzed, and the results demonstrated that miR-769-3p exhibited a significant 
association with the WHO grade and KPS score. In addition, Cox regression analysis results confirmed that miR-769-3p levels and the WHO grade were independent prognostic factors for glioma. These results suggested a potential prognostic value for miR-769-3p in glioma; therefore, miR-769-3p may be a novel diagnostic and prognostic factor for glioma.To further explore the functional role of miR-769-3p in glioma, MTT and Transwell in vitro assays were performed. The results demonstrated that overexpression of miR-769-3p inhibited cell proliferation, migration and invasion in glioma cells, suggesting that miR-769-3p may act as a tumor suppressor and inhibit glioma growth and metastasis.

ZEB2, also termed SIP1, is a member of zinc-finger E-box binding proteins (27). ZEB2 is located in the nucleus and is reported to serve as a DNA-binding transcriptional repressor by interacting with activated Smad proteins (28). A previous study has suggested a promoting role of ZEB2 in epithelial-mesenchymal transition, which is involved in tumor metastasis (29). In the present study, an important molecular link was observed between miR-769-3p and ZEB2, as miR-769-3p negatively regulated the level of ZEB2 in glioma cells. Previous studies have demonstrated that aberrant expression of ZEB2 is associated with the development and progression of various types of cancer, such as gastric, breast and liver cancer (30-32). In addition, upregulation of ZEB2 has been detected in patients with glioma, and ZEB2 has been demonstrated to effectively promote tumor cell progression (33,34). Collectively, these data supported the hypothesis of the present study that ZEB2 was a direct target gene of miR-769-3p in glioma.

The Wnt $/ \beta$-catenin pathway is considered to be one of the most important molecular pathways involved in the development of various types of human cancer, including glioma $(35,36)$. Downregulation of ZEB2 reduces cell proliferation and suppresses the expression of $\beta$-catenin, c-Myc and cyclin D1 in glioma, suggesting the involvement of ZEB2 in the regulation of the Wnt/ $\beta$-catenin pathway in patients with glioma (34). Therefore, the present study focused on the association between miR-769-3p and the Wnt/ $\beta$-catenin pathway, and the results suggested that overexpression of miR-769-3p suppressed the expression levels of Wnt signaling-related proteins. Thus, the results of the present study indicated that miR-769-3p inhibited glioma cell progression by targeting ZEB2 and further affecting the $\mathrm{Wnt} / \beta$-catenin signaling pathway.

In conclusion, the results of the present study suggested a crucial role for miR-769-3p in the occurrence and development of glioma. These results indicate a potential clinical value of miR-769-3p as an effective biomarker for the diagnosis and prognosis of glioma. In addition, the results of the present study demonstrated that miR-769-3p exerted its tumor suppressor role by targeting ZEB2 and inhibiting the $\mathrm{Wnt} / \beta$-catenin signaling pathway. Therefore, miR-769-3p may be a novel therapeutic target for the treatment of glioma.

\section{Acknowledgements}

Not applicable.

\section{Funding}

No funding was received.

\section{Availability of data and materials}

The datasets used and/or analyzed during the current study are available from the corresponding author on reasonable request.

\section{Authors' contributions}

$\mathrm{KW}$ and QS initiated and designed this work and were responsible for the data acquisition, data analysis and writing the manuscript. SY and YG collected clinical tissues, performed RNA extraction and RT-qPCR and data analysis. CZ performed the in vitro experiments.

\section{Ethics approval and consent to participate}

This study was completed with the approval of the Ethics Committee of Weihai Central Hospital, and written informed consent was collected from each patient.

\section{Patient consent for publication}

Not applicable.

\section{Competing interests}

The authors declare that they have no competing interests.

\section{References}

1. Liang J, Zhang XL, Li S, Xie S, Wang WF and Yu RT: Ubiquitin-specific protease 22 promotes the proliferation, migration and invasion of glioma cells. Cancer Biomark 23: 381-389, 2018.

2. Zhao W, Yin CY, Jiang J, Kong $\mathrm{W}, \mathrm{Xu} \mathrm{H}$ and Zhang $\mathrm{H}$ : MicroRNA-153 suppresses cell invasion by targeting SNAI1 and predicts patient prognosis in glioma. Oncol Lett 17: 1189-1195, 2019.

3. Yang D, Yuan Y, Zhang S, Zhao K, Li F, Ren H, Zhang Z and Yu Y: Association between IL-13 Gene rs20541 polymorphism and glioma susceptibility: A meta-analysis. Oncol Res Treat 41: 14-21, 2018.

4. Hu S, Xu L, Li L, Luo D, Zhao H, Li D and Peng B: Overexpression of lncRNA PTENP1 suppresses glioma cell proliferation and metastasis in vitro. Onco Targets Ther 12: 147-156, 2019.

5. Zhang Z, Huang X, Li J, Fan H, Yang F, Zhang R, Yang Y, Feng S, He D, Sun W and Xin T: Interleukin 10 promotes growth and invasion of glioma cells by up-regulating KPNA 2 in vitro. J Cancer Res Ther 15: 927-932, 2019.

6. Hao B, Chen X and Cao Y: Yes-associated protein 1 promotes the metastasis of U251 glioma cells by upregulating Jagged-1 expression and activating the Notch signal pathway. Exp Ther Med 16: 1411-1416, 2018.

7. Jiang YR, Du JY, Wang DD and Yang X: miRNA-130a improves cardiac function by down-regulating TNF- $\alpha$ expression in a rat model of heart failure. Eur Rev Med Pharmacol Sci 22: 8454-8461, 2018.

8. Wu YF, Ou CC, Chien PJ, Chang HY, Ko JL and Wang BY: Chidamide-induced ROS accumulation and miR-129-3p-dependent cell cycle arrest in non-small lung cancer cells. Phytomedicine 56: 94-102, 2018.

9. Chen X and Wang A: Clinical significance of miR-195 in hepatocellular carcinoma and its biological function in tumor progression. Onco Targets Ther 12: 527-534, 2019.

10. Huang S, Zheng S, Cheng H, Lin Y, Wen Y and Lin W: Flot2 targeted by miR-449 acts as a prognostic biomarker in glioma. Artif Cells Nanomed Biotechnol 47: 250-255, 2019.

11. Lu GF, Geng F, Xiao Z, Chen YS, Han Y, You CY, Gong NL, Xie ZM and Pan M: MicroRNA-6807-3p promotes the tumorigenesis of glioma by targeting downstream DACH1. Brain Res 1708: 47-57, 2019. 
12. Liu F, Xiong Y, Zhao Y, Tao L, Zhang Z, Zhang H, Liu Y, Feng G, $\mathrm{Li} \mathrm{B}, \mathrm{He} \mathrm{L}$, et al: Identification of aberrant microRNA expression pattern in pediatric gliomas by microarray. Diagn Pathol 8: 158, 2013.

13. Han C, Song Y and Lian C: MiR-769 inhibits colorectal cancer cell proliferation and invasion by targeting HEY1. Med Sci Monit 24: 9232-9239, 2018.

14. Zhang CJ and Du HJ: Screening key miRNAs for human hepatocellular carcinoma based on miRNA-mRNA functional synergistic network. Neoplasma 64: 816-823, 2017.

15. Ju Q, Zhao L, Gao J, Zhou L, Xu Y, Sun Y and Zhao X: Mutant p53 increases exosome-mediated transfer of miR-21-3p and miR-769-3p to promote pulmonary metastasis. Chin J Cancer Res 31: 533-546, 2019.

16. Livak KJ and Schmittgen TD: Analysis of relative gene expression data using real-time quantitative PCR and the 2(-Delta Delta $\mathrm{C}(\mathrm{T}))$ method. Methods 25: 402-408, 2001.

17. Komori T: The 2016 WHO classification of tumours of the central nervous system: The major points of revision. Neurol Med Chir (Tokyo) 57: 301-311, 2017.

18. Zhang F, Wu A, Wang Y and Liu J: miR-490-3p functions as a tumor suppressor in glioma by inhibiting high-mobility group AT-hook 2 expression. Exp Ther Med 18: 664-670, 2019.

19. Ostrom QT, Bauchet L, Davis FG, Deltour I, Fisher JL, Langer CE, Pekmezci M, Schwartzbaum JA, Turner MC, Walsh KM, et al: The epidemiology of glioma in adults: A 'state of the science' review. Neuro Oncol 16: 896-913, 2014.

20. Chung DS, Shin HJ and Hong YK: A new hope in immunotherapy for malignant gliomas: Adoptive T cell transfer therapy. J Immunol Res 2014: 326545, 2014.

21. Waghmare I, Roebke A, Minata M, Kango-Singh M and Nakano I: Intercellular cooperation and competition in brain cancers: Lessons from Drosophila and human studies. Stem Cells Transl Med 3: 1262-1268, 2014.

22. Lima FR, Kahn SA, Soletti RC, Biasoli D, Alves T, da Fonseca AC Garcia C, Romao L, Brito J, Holanda-Afonso R, et al: Glioblastoma: Therapeutic challenges, what lies ahead. Biochim Biophys Acta 1826: 338-349, 2012.

23. Wang R, Zuo X, Wang K, Han Q, Zuo J, Ni H, Liu W, Bao H, Tu Y and Xie P: MicroRNA-485-5p attenuates cell proliferation in glioma by directly targeting paired box 3 . Am J Cancer Res 8: 2507-2517, 2018.

24. Wang LQ, Sun W, Wang Y, Li D and Hu AM: Downregulation of plasma miR-124 expression is a predictive biomarker for prognosis of glioma. Eur Rev Med Pharmacol Sci 23: 271-276, 2019.

25. Qiu HJ, Lu XH, Yang SS, Weng CY, Zhang EK and Chen FC: MiR-769 promoted cell proliferation in human melanoma by suppressing GSK3B expression. Biomed Pharmacother 82: 117-123, 2016.
26. Zhang W, Zhao W, Ge C, Li X, Yang X, Xiang Y and Sun Z: Decreased let-7b is associated with poor prognosis in glioma. Medicine (Baltimore) 98: e15784, 2019.

27. Epifanova E, Babaev A, Newman AG and Tarabykin V: Role of Zeb2/Sip1 in neuronal development. Brain Res 1705: 24-31, 2019.

28. Li Y, Zhang J, Zhang W, Liu Y, Wang K, Zhang Y, Yang C, Li X, Shi J, Su L and Hu D: MicroRNA-192 regulates hypertrophic scar fibrosis by targeting SIP1. J Mol Histol 48: 357-366, 2017.

29. Ji H, Sang M, Liu F, Ai N and Geng C: miR-124 regulates EMT based on ZEB2 target to inhibit invasion and metastasis in triple-negative breast cancer. Pathol Res Pract 215: 697-704 2019.

30. Li H, Xu L, Zhao L, Ma Y, Zhu Z, Liu Y and Qu X: Insulin-like growth factor-I induces epithelial to mesenchymal transition via GSK-3ßand ZEB2 in the BGC-823 gastric cancer cell line. Oncol Lett 9: 143-148, 2015.

31. Zou Y, Ouyang Q, Wei W, Yang S, Zhang Y and Yang W: FAT10 promotes the invasion and migration of breast cancer cell through stabilization of ZEB2. Biochem Biophys Res Commun 506: 563-570, 2018.

32. Zhang X, Xu X, Ge G, Zang X, Shao M, Zou S, Zhang Y, Mao Z, Zhang J, Mao F, et al: miR498 inhibits the growth and metastasis of liver cancer by targeting ZEB2. Oncol Rep 41: 1638-1648, 2019.

33. Suzuki K, Kawataki T, Endo K, Miyazawa K, Kinouchi H and Saitoh M: Expression of ZEBs in gliomas is associated with invasive properties and histopathological grade. Oncol Lett 16: 1758-1764, 2018.

34. Qi S, Song Y, Peng Y, Wang H, Long H, Yu X, Li Z, Fang L, Wu A, Luo W, et al: ZEB2 mediates multiple pathways regulating cell proliferation, migration, invasion, and apoptosis in glioma. PLoS One 7: e38842, 2012.

35. Gong X, Liao X and Huang M: LncRNA CASC7 inhibits the progression of glioma via regulating Wnt $/ \beta$-catenin signaling pathway. Pathol Res Pract 215: 564-570, 2019.

36. Wang Y, Yang Q, Cheng Y, Gao M, Kuang L and Wang C: Myosin heavy chain 10 (MYH10) gene silencing reduces cell migration and invasion in the glioma cell lines U251, T98G, and SHG44 by inhibiting the Wnt/ $\beta$-catenin pathway. Med Sci Monit 24: 9110-9119, 2018.

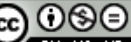

This work is licensed under a Creative Commons Attribution-NonCommercial-NoDerivatives 4.0 International (CC BY-NC-ND 4.0) License. 